\title{
Anopheles gambiae heat shock protein cognate 70B impedes o'nyong-nyong virus replication Cheolho Sim ${ }^{1,4}$, Young S Hong2, Konstantin A Tsetsarkin ${ }^{3}$, Dana L Vanlandingham ${ }^{3}$, Stephen Higgs ${ }^{3}$ and Frank H Collins*1
}

\author{
Address: ${ }^{1}$ The Center for Global Health and Infectious Diseases, University of Notre Dame, Notre Dame, IN 46556, USA, ${ }^{2}$ Department of Tropical \\ Medicine, Tulane University, 1430 Tulane Avenue SL-17, New Orleans, LA 70112, USA, 3Pathology Department, Keiller Bldg., 301 University \\ Blvd., University of Texas Medical Branch, Galveston, TX 77555-0609, USA and ${ }^{4}$ Department of Entomology, The Ohio State University, \\ Columbus, Ohio 43210, USA \\ Email: Cheolho Sim - sim.15@osu.edu; Young S Hong - young@tulane.edu; Konstantin A Tsetsarkin - kotsetsa@utmb.edu; \\ Dana L Vanlandingham - dlvanlan@utmb.edu; Stephen Higgs - sthiggs@utmb.edu; Frank H Collins* - frank@nd.edu \\ * Corresponding author
}

Published: II July 2007

BMC Genomics 2007, 8:231 doi:10.1|86/147|-2164-8-231
Received: 22 March 2007

Accepted: II July 2007

This article is available from: http://www.biomedcentral.com/|47|-2164/8/23I

(C) 2007 Sim et al; licensee BioMed Central Ltd.

This is an Open Access article distributed under the terms of the Creative Commons Attribution License (http://creativecommons.org/licenses/by/2.0), which permits unrestricted use, distribution, and reproduction in any medium, provided the original work is properly cited.

\begin{abstract}
Background: Phylogenetic and functional analysis was conducted on an Anopheles gambiae gene, ENSANGG000000 17398. Based on phylogenetic analysis, this gene belongs to the same lineage as Heat shock protein cognate 70-4 (Hsc70-4) in Drosophila. Accordingly, we propose to name this gene Heat shock protein cognate 70B (HSC70B). We previously reported that expression of $\mathrm{HSC70B}$ and other genes including elongation factor- $\mathrm{I} \alpha(\mathrm{EF}-\mathrm{I} \alpha)$ and the agglutinin attachment subunit (agglutinin) were up-regulated in o'nyong-nyong virus (ONNV)-infected female An. gambiae. Double-stranded RNA interferences have been applied to further investigate HSC70B, EF-I $\alpha$ and the agglutinin functions in ONNV replication in An. gambiae.
\end{abstract}

Results: Among these three RNAi silenced genes, only dsRNAs of HSC70B (dsHSC70B) promoted ONNV replication in adult An. gambiae compared to the control mosquitoes that were co-injected with ONNV and dsRNA of $\beta$-galactosidase (ds $\beta$-gal). ONNV titers from mosquitoes co-injected with dsHSC70B were about 9-fold higher at 6 days post-injection (d.p.i.) as compared to the control mosquitoes. By using ONNV tagged with enhanced green fluorescent protein (ONNV-eGFP), co-injection of ONNV-eGFP with dsHSC7OB also showed approximately $2 \sim 3$ fold higher GFP expression rates than the controls in the head, thorax, and abdomen of the mosquito. Furthermore, co-injection of ONNV with dsHSC70B significantly reduced the lifespan of adult mosquitoes as compared with the control, co-injection of ONNV with ds $\beta$-gal treated mosquitoes.

Conclusion: These results indicate that HSC70B plays important roles in homeostasis and suppression of ONNV replication in the vector, An. gambiae. Biological implications of these findings are that while mosquitoes allow ONNV to replicate in them, they also check viral titers so that ONNV infection will result in no harmful effect on mosquitoes. Therefore, mosquitoes can function as vectors of ONNV transmission to humans while ONNV infection in An. gambiae remains asymptomatic. 


\section{Background}

The arbovirus, o'nyong-nyong virus (ONNV) belongs to genus Alphavirus, and is an enveloped, single stranded, (+) RNA virus with a genome of approximately $12 \mathrm{~kb}$ $[1,2]$. Unlike other arboviruses, ONNV is primarily transmitted by anopheline mosquitoes such as Anopheles gambiae and An. funestus [3]. ONNV was first identified during an epidemic in Uganda in 1959, which ultimately infected over 2 million people across East Africa from 1959 to 1961 [4]. Recently it has reemerged in 1996 and 2003 sporadically in Africa $[5,6]$.

Although mosquitoes are critical vectors in many arboviral transmission cycles, there is limited information on how arboviruses influence mosquito gene expression and how mosquito immune systems defend arthropod vectors from deleterious consequences of viral infection. The recent completion of the sequencing of the An. gambiae genome has allowed us to investigate modulation of mosquito gene expression resulting from arbovirus infection. Genome-wide screening of differentially expressed transcripts of ONNV-infected female An. gambiae relative to naïve females was conducted at 14 day p.i. [7]. Seven genes were identified for their differential expression in ONNV-infected An. gambiae compared with controls by cDNA microarrays followed by paired t-test and quantitative real time PCR (qRT-PCR) analysis. The products of the seven genes are seemingly involved in protein translation, DNA replication, or intracellular transport pathways [1]. Among the seven candidates, HSC70B, EF- $1 \alpha$ and agglutinin were chosen for further functional studies because of their roles in protein folding, protein elongation, cell adhesion, and cytoprotection, which are all important molecular processes for viral replication.

We hypothesize that elevated gene expression of HSC70B for example, may protect the mosquito cells from ONNVinduced molecular damage [8]. Since molecular chaperons including heat shock protein 70 families regulate protein folding and degradation, it is possible that HSC70B may suppress the non-native viral structural or non-structural protein synthesis in mosquito cells. It was also reported that mammalian and mosquito EF- $1 \alpha$ binds to the 3' UTRs of West Nile virus (WNV) and a range of RNA viruses [9-12]. Thus, Anopheles EF-1 $\alpha$ may have a similar role for ONNV replication in An. gambiae. Lastly, agglutinin is a membrane attachment subunit that may interact with ONNV on the membranes of endosomes and lysosomes. Because non-structural proteins and RNAs of alphaviruses including ONNV are associated with the membranes of modified endosomes and lysosomes in the replication complex [13], agglutinin may therefore be involve in a membrane attachment of the replication complex of ONNV.
Based on these assumptions, HSC70B, EF- $1 \alpha$ and agglutinin genes were subjected to a detailed functional analysis for their potential involvement in ONNV replication. Using RNAi, we post-transcriptionally silenced target transcripts of the three genes, HSC70B, EF-1 $\alpha$ and agglutinin by co-injecting dsRNAs of each target transcript with ONNV into female An. gambiae. The result showed that silencing the HSC70B transcript caused significant increase of ONNV titers in female mosquitoes whilst the remaining two genes had no noticeable effects. Herein, we discuss potential antiviral activity of HSC70B in An. gambiae.

\section{Results}

Phylogenetic analysis of $70 \mathrm{Kda}$ Heat shock protein family from An. gambiae and D. melanogaster

Phylogenetic analysis of HSP70 genes from An. gambiae and D. melanogaster indicate that Anopheles HSC70B gene is evolutionarily more conserved with Drosophila Hsc701 and Hsc70-4 genes than other Anopheles homologues (Fig 1.). For example, Anopheles HSC70B is most tightly liked to Drosophila Hsc70-4 (Fig. 1). Multiple sequence alignments indicated that there are high polymorphisms near the 5' and 3'-end coding and non-coding regions of the Anopheles HSC70 family (Fig. 2). Indeed, based on these sequence polymorphisms among the Anopheles HSC70 family, we were able to detect unique transcripts of the Anopheles HSC70B gene among the Anopheles HSP70 genes by using the RT-PCR and qRT-PCR primers based on the 3 'and 5'-end regions, respectively (Fig. 3 and Table 1). In addition, the dsRNA of Anopheles HSC70B was designed based on the $5^{\prime}$ end that was specific to the HSC70B gene, which enabled HSC70B specific knockdown in Anopheles gambiae.

\section{The effect of HSC7OB on ONNV replication}

First, efficiency of dsRNAi was assessed by using semiquantitative RT-PCR and qRT-PCR analyses. In contrast to high induction of HSC70B in ds $\beta$-gal and ONNV/ONNVeGFP coinjected mosquitoes, only traces of HSC70B mRNA were detected in dsHSC70B mosquitoes using semi-quantitative RT-PCR and primers corresponding to the 3 ' end of the HSC70B gene (Fig. 3A). This result shows that the injection of specific dsHSC70B successfully reduced endogenous target transcripts of $\mathrm{HSC70B}$ gene after ONNV injection. Similarly, RT-PCR results also showed the successful post-transcriptional inhibition in agglutinin and EF-1 $\alpha$ genes (Fig. 3A).

Mosquitoes were coinjected with $\sim 3.1 \times 10^{2}$ pfu of virus and $625 \mathrm{ng}$ of the respective dsRNAs targeting the HSC70B, agglutinin and EF- $1 \alpha$ gene or the $\beta$-gal gene as an internal control (Table 1). To quantify ONNV in the respective dsRNAi-treated mosquitoes, each An. gambiae was triturated and ONNV titer was quantified by plaque 
Table I: The primer list of qRT-PCR, RT-PCR, and dsRNAs template

\begin{tabular}{|c|c|c|c|}
\hline Gene ID (GenBank Accession No.) & Primer sequence ( $5^{\prime}$ to $\left.3^{\prime}\right)$ & Product size (bp) & Amplification efficiencyc $\left(R^{d}\right)$ \\
\hline${ }^{\mathrm{a} q R p S 4}(\underline{\mathrm{A} J 283756})$ & $\begin{array}{l}\text { Forward: GCTGCCGCTGGTGATCTT } \\
\text { Reverse: TCGTCACCTCGCTGTTGGT }\end{array}$ & 65 & $0.90(0.99)$ \\
\hline 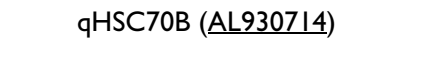 & $\begin{array}{l}\text { Forward: GCGATCCAGGCCGACAT } \\
\text { Reverse: TCTTTGGCTTGCCCTCGAT }\end{array}$ & 64 & $0.97(0.99)$ \\
\hline EF-I $\alpha(\underline{X M 308429.1})$ & $\begin{array}{l}\text { Forward: CGAGAAGGAAGCTCAGGAGA } \\
\text { Reverse: ACGACGACACCTCCTTCTTG }\end{array}$ & 404 & \\
\hline agglutinin (XM3||465.2) & $\begin{array}{l}\text { Forward: CGGGCGAAACTTACTACAGC } \\
\text { Reverse: CGATGGCTACGTTACGGAAT }\end{array}$ & 435 & \\
\hline HSC70B (AL9307|4 $)$ & $\begin{array}{l}\text { Forward: GTGAACGAGGCCGAGAAGTA } \\
\text { Reverse: TAGTCGACCTCCTCGATGGT }\end{array}$ & 410 & \\
\hline RpS7 (XM 314557.2) & $\begin{array}{l}\text { Forward: ATCGCTATGGTGTTCGGTTC } \\
\text { Reverse: GCTGCAAACTTCGGCTATTC }\end{array}$ & 627 & \\
\hline EI (AF079456.I) & $\begin{array}{l}\text { Forward: AGAGCCCACACAGCTTCAGT } \\
\text { Reverse: ACCGGGTTTGTTGCTATCTG }\end{array}$ & 431 & \\
\hline NSI (AF079456.I) & $\begin{array}{l}\text { Forward: CTTCCTGATGTGCAAGACGA } \\
\text { Reverse: ACGACCACAGGCTTGGTATC }\end{array}$ & 440 & \\
\hline bdsEF-I $\alpha(\underline{\text { XM308429.I }})$ & $\begin{array}{l}\text { Forward: TAATACGACTCACTATAGGCG } \\
\text { AGAAGGAAGCTCAGGAGA } \\
\text { Reverse: TAATACGACTCACTATAGGAC } \\
\text { GACGACACCTCCTTCTTG }\end{array}$ & 442 & \\
\hline ds-agglutinin ( $\underline{\mathrm{X}} 33|| 465.2)$ & $\begin{array}{l}\text { Forward: TAATACGACTCACTATAGGCG } \\
\text { GGCGAAACTTACTACAGC } \\
\text { Reverse: TAATACGACTCACTATAGGCG } \\
\text { ATGGCTACGTTACGGAAT }\end{array}$ & 473 & \\
\hline dsHSC70B (AL9307|4 $)$ & $\begin{array}{l}\text { Forward: TAATACGACTCACTATAGGTT } \\
\text { ACGGTGCGAGCAGAAAAT } \\
\text { Reverse: TAATACGACTCACTATAGGCA } \\
\text { CGTTCAGTCCCGAGATGT }\end{array}$ & 652 & \\
\hline ds $\beta$-gal ( & $\begin{array}{l}\text { Forward: TAATACGACTCACTATAGGGG } \\
\text { TCGCCAGCGGCACCGCGCCTTTC } \\
\text { Reverse: TAATACGACTCACTATAGGGC } \\
\text { CGGTAGCCAGCGCGGATCATCGG }\end{array}$ & 545 & \\
\hline
\end{tabular}

\footnotetext{
${ }^{a} \mathrm{q}$ represent the primer pairs for quantitative RT-PCR, bds represents the primer pairs of the templates for dsRNA includes T7 promoter for in vitro transcription. 'Amplification efficiencies were calculated from the slope of standard curves as $E=10[-1 / s l o p e]-I$. I00\% PCR efficiency corresponds to an amplification efficiency of I (Applied Biosystems Application Note); dRegression coefficient of linear standard curve.
}

assay from the treatment groups. Mosquitoes coinjected with ONNV and dsHSC70B had significantly more plaques than those mosquitoes coinjected with ONNV and ds $\beta$-gal $(P=0.00045)$ at 6 d.p.i. (Fig. 4). No significant differences in the number of plaques were observed among the mosquitoes injected with ONNV and each of ds-agglutinin, dsEF-1 $\alpha$, and ds $\beta$-gal ( $P \geq 0.74)$ (Fig. 4).

To investigate whether up-regulation of HSC70B gene impedes ONNV replication, An. gambiae was infected with eGFP-tagged ONNV whilst HSC70B transcripts were silenced by dsRNAi. ONNV titers were then indirectly estimated by visually comparing eGFP expression among three mosquito groups that were coinoculated with dsHSC70B, or ds $\beta$-gal or buffer. In control mosquitoes injected with ds $\beta$-gal or buffer, expression of eGFP was usually weak in head, thorax and abdomen tissues (Fig. 5 and Table 2). Mosquitoes coinjected with ONNV-eGFP and dsHSC70B typically had stronger expression of eGFP in all three tissues (Fig. 5). At 6 dpi, $47 \%(n=32)$ of mosquitoes receiving ds $\beta$-gal expressed eGFP in thoracic tis- sues. However, $87 \%(n=23)$ expressed eGFP in thoracic tissues when dsHSC70B was silenced (Table 2). In a similar way, the mosquitoes receiving ds $\beta$-gal showed 38\% ( $n$ $=32)$ and $22 \%(n=32)$ of eGFP expression in head and abdomen, respectively (Table 2 ). In contrast, mosquitoes receiving dsHSC70B showed 70\% $(n=23)$ and $65 \%(\mathrm{n}=$ 23 ) of eGFP expression in head and abdomen, respectively (Table 2).

The ribosomal proteins S4 (RpS4) and S7 (RpS7) were used as internal controls for infection studies of ONNV and the malaria parasite, Plasmodium berghei, respectively $[7,14]$. When RpS7 was analyzed by qRT-PCR and cDNA microarray studies, Student's t test determined that the mRNA expression levels of RpS7 in uninfected and ONNV-infected mosquitoes collected at $24 \mathrm{~h}, 48 \mathrm{~h}$ and 14 days p.i. were not significantly different (data not shown). As shown in Figure 3, RT-PCR analysis of RpS7 and qRTPCR analysis of RpS4 transcript levels at 6 days p.i., detected no significant difference between the relative mRNA levels derived from treated mosquitoes. The results 


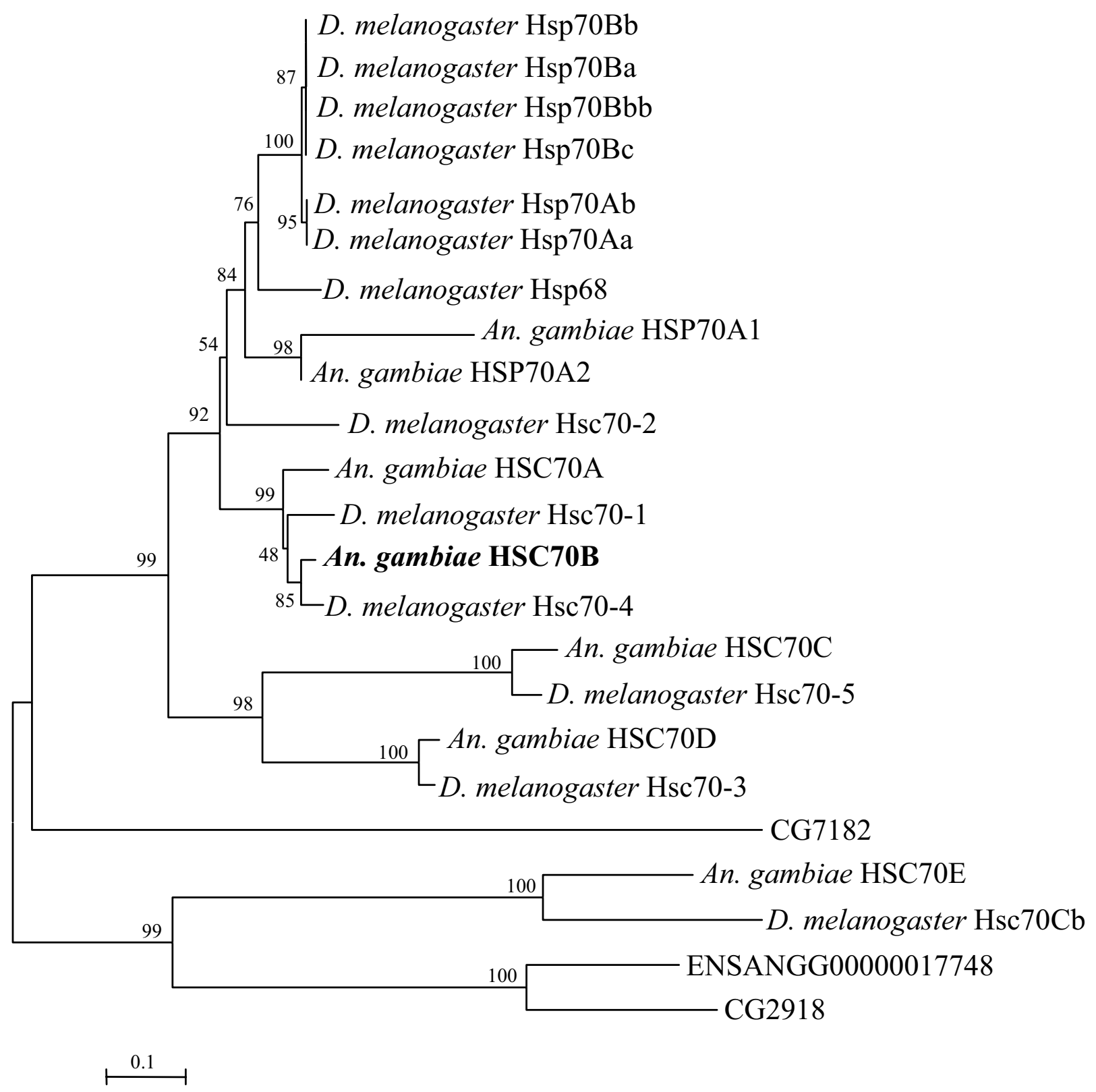

\section{Figure I}

Phylogenetic tree and multiple sequence alignment of the HSP70 family from An. gambiae and D. melanogaster. The numbers for interior branches represent bootstrap values. The scale bar indicates an evolutionary distance of 0.1 amino acid substitutions per position. Accession numbers of the nucleotide and amino acid sequences used: An. gambiae: HSP70AI, ENSANGG0000000 I 248; HSP70A2, ENSANGG00000022650; HSC70A, ENSANGG00000019768; HSC70B, ENSANGG000000 17398; HSC70C, ENSANGG000000 16503; HSC70D, ENSANGG000000 10404; HSC70E, ENSANGG000000 I 2804; ENSANGG000000 I7748. D. melanogaster: Hsp70Bb, CG3 I 359; Hsp70Bbb, CG5834; Hsp70Bc, CG6489; Hsp70Bb, CG31359; Hsp70Ab, CG 18743; Hsp70Aa, CG3 I366; Hsp68, CG5436; Hsc70-2, CG7756; Hsc70-I, CG8937; Hsc70-4, CG4264; Hsc70-5, CG8542; Hsc70-3, CG4I47; CG7I82; Hsc70Cb, CG6603; CG29I8. 


\begin{tabular}{|c|c|}
\hline HSC70B & - \\
\hline HSC7OD & 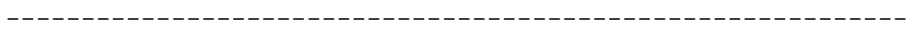 \\
\hline $\mathrm{HSC} 70 \mathrm{C}$ & 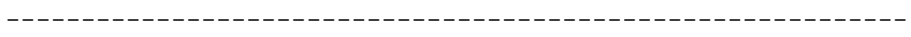 \\
\hline HSC 70E & AGCGTTTGCACCTATACTGTGCCTGAAATCAGATCATTAATTCGCGTGGGAACTTTGGTT \\
\hline $\mathrm{HSC} 70 \mathrm{~A}$ & - \\
\hline HSC70B & 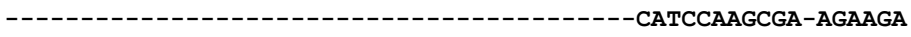 \\
\hline HSC70D & 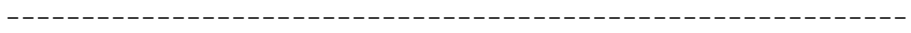 \\
\hline $\mathrm{HSC} 70 \mathrm{C}$ & ------ -- -- -- - - - - - - - - - - - - - - - - - - - - - - - - - - - - - - - - - - - - \\
\hline $\mathrm{HSC} 70 \mathrm{E}$ & CATCTGTGAAACTGAGCAAGAGTGGCAACTAGAATAGTTCTCTGAGATTTAATCAACTTA \\
\hline HSC 70A & ------------------------------------------ CAGCGAACGGACAGCACG \\
\hline HSC70B & AGCAGACGGTGCGAGCA-GAAAATCGCGAGAAGTTCGTTCCAAGTGCAAGTCAGTGACCA \\
\hline HSC 70D & --------------ATGAAGCTATTGAAACAGACGGCCCTGGTGGTCGT-GCTGGCCG \\
\hline $\mathrm{HSC} 70 \mathrm{C}$ & 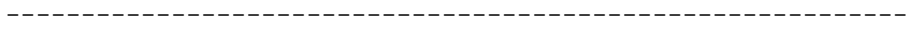 \\
\hline $\mathrm{HSC} 70 \mathrm{E}$ & TTTCCAGATTCTGAAAATTTCTTCCGCTGTTGTGAAACTTCCTTTGGCGGCGCCGAGTCG \\
\hline HSC70A & TTCAGCAACAAGGTGTAAGAAAACCACGTGCAGTAAATCGGTGACATTGTTCTTTAGCCG \\
\hline HSC70B & GTAAATCGGAAAAAACACATTATAATCAAAGATGGCAGCCGCAAAAGCAC--CAGCAGTC \\
\hline HSC70D & TGTTGGCCTGTTCGGCGGAGGAAAAGAAGGAAAAGGACAAGGATATCGGCA-CGGTGGTC \\
\hline HSC $70 \mathrm{C}$ & ----------------------------------- CAGGTCAAGGGTG-CAGTTATC \\
\hline $\mathrm{HSC} 70 \mathrm{E}$ & AAGAAAAATTGTAATAAAATCCAACGAGATAATCCAGTAAACCTCAAAATGTCAGTGATT \\
\hline HSC70A & TACAAAAAGATAAGACCGATCACAC---AGGTCAGAAATGGCAAAGACAC--CAGCTGTC \\
\hline HSC70B & GGTATTGATCTGGGTACGACCTACAGCTGTGTCGGAGTGTTCCAGCACGGCAAGGTCGAG \\
\hline HSC7OD & GGTATCGATCTCGGCACCACCTACTCCTGCGTCGGTGTGTACAAGAACGGGCGCGTGGAA \\
\hline $\mathrm{HSC} 70 \mathrm{C}$ & GGTATCGATCTCGGCACCACCAACTCGTGCGTCGCCGTGATGGAGGGCAAAAATGCCAAA \\
\hline HSC 70E & GGAATTGATTTTGGAAATGATTCGTCATATGTGGCGGTCGCCAAAGCAGGAGGCATCGAG \\
\hline HSC 70A & GGTATAGATCTTGGCACTACCTACTCGTGCGTCGGTGTGTTCCAGCATGGCAAGGTGGAC \\
\hline & 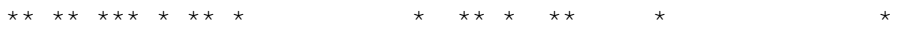 \\
\hline HSC70B & АTCATCGCCAACGACCAGGGCAACCGAACCACGCCATCGTATGTGGCCTTCAC---TGAT \\
\hline HSC 70D & ATCATTGCCAACGACCAGGGTAACCGCATCACGCCGTCCTACGTGGCATTCACGGCCGAC \\
\hline $\mathrm{HSC} 70 \mathrm{C}$ & GTCATCGAGAATGCGGAGGGCGCCCGTACCACCCCGTCCCATGTGGCGTTCACCAAGGAC \\
\hline $\mathrm{HSC} 70 \mathrm{E}$ & ACCATCGCCAACGACTACAGTTTGCGGGCCACACCATCGTTCGTGGCATTCGC---AGGA \\
\hline HSC 70A & ATTATCGCGAACGATCAGGGCAACCGGACCACCCCGTCGTACGTGGCGTTCAC---TGAT \\
\hline & 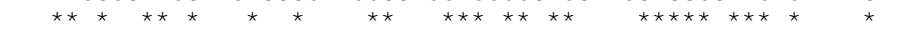 \\
\hline HSC70B & ACGGAGCGTCTGATTGGCGATGCTGCCAAGAACCAGGTGGCCATGAACCCGACCAACACC \\
\hline HSC70D & GGTGAGCGTCTGATCGGAGACGCCGCCAAGAACCAGCTGACGACCAATCCGGAGAACACC \\
\hline $\mathrm{HSC} 70 \mathrm{C}$ & GGCGAACGGTTGGTGGGCATGCCGGCCAAACGCCAGGCCGTCACCAACTCAGCCAACACA \\
\hline HSC 70E & CGTAATCGTGTACTTGGTGTTGCTGCCAAAAATCAACAAGTCACTAACATGAACAATACT \\
\hline HSC70A & TCGGAACGGTTGATTGGAGATGCTGCCAAGAACCAGGTGGCGATGAATCCCAACAACACC \\
\hline & 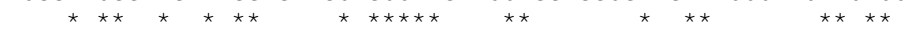 \\
\hline HSC70B & АTCTTCGACGCGAAGCGTCTGATCGGCCGCAAGTTCGACGATCCCGCGATCCAGGCCGAC \\
\hline HSC 70D & GTGTTCGATGCGAAGCGTCTGATTGGCCGCGAGTTCACCGACCACACGGTGCAGCACGAC \\
\hline $\mathrm{HSC} 70 \mathrm{C}$ & TTCTACGCCACCAAGCGACTGATCGGCCGCCGGTTCGATGATGCCGAAATCAAGAAAGAT \\
\hline $\mathrm{HSC} 70 \mathrm{E}$ & ATTGGCAACTTTAAGGAGTTGCTTGGCCGTAAGTTCGACGATCCTCGTGCGCAGGAAGAA \\
\hline HSC70A & ATTTTTGATGCCAAACGCTTGATCGGTCGCAAGTTTGACGATGCCACAGTACAGGCCGAC \\
\hline & 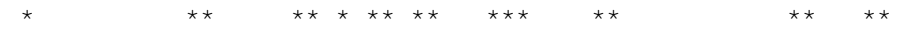 \\
\hline HSC70B & АTGAAGCACTGGCCGTTCGA---GGTGGAGTCGATCGAGGGCAAGCCAAAGATCGCGGTC \\
\hline HSC70D & ATCAAGCTGCTGCCGTTCAA---GGTGATCGAAAAGAACTCCAAGCCGCACATCAGGGTG \\
\hline $\mathrm{HSC} 70 \mathrm{C}$ & CTCGCCAACCTGTCCTACAA---GGTGGT----GAAGGCGTCGAACGGTGACGCGTGG-- \\
\hline HSC 70E & СTTCGTTCATTACCGTACCATACGGAGGCTTTACAAGATGGTGGTATCGGAATAAGAGTC \\
\hline HSC 70A & ATGAAGCACTGGCCATTCGA---TGTGATCAGTGACGGCGGCAAACCGAAGCTGCAGGTC \\
\hline
\end{tabular}

Figure 2

Multiple sequence alignment of $\mathbf{5}^{\prime}$ end of coding and non-coding regions of HSC70 family. The arrows and red characters represent the highly polymorphic sites for the qRT-PCR primers. The bold characters represent the HSC70B gene and the DNA template for the dsRNA of HSC70B. The asterisks denote the conserved sequences among the HSC70 gene family. Accession numbers of the nucleotide and amino acid sequences used: An. gambiae: HSC70A, ENSANGG000000 I9768; HSC70B, ENSANGG000000 I7398; HSC70C, ENSANGG00000016503; HSC70D, ENSANGG000000 I 0404; HSC70E, ENSANGG000000 I 2804. 

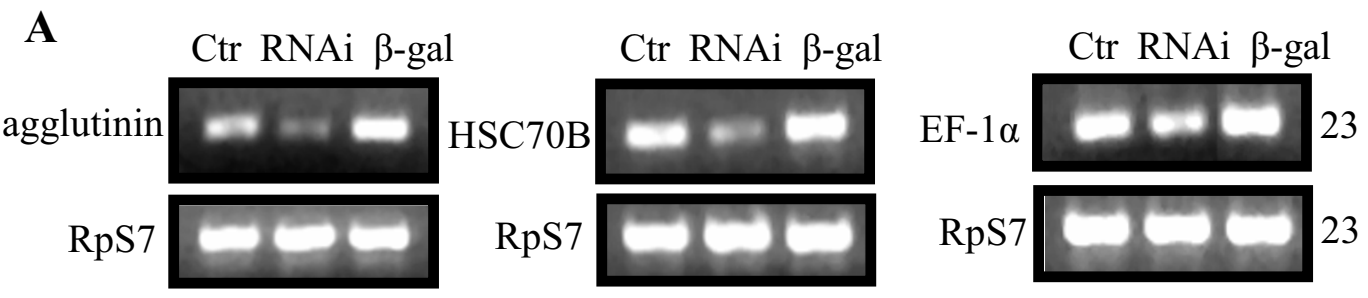

B

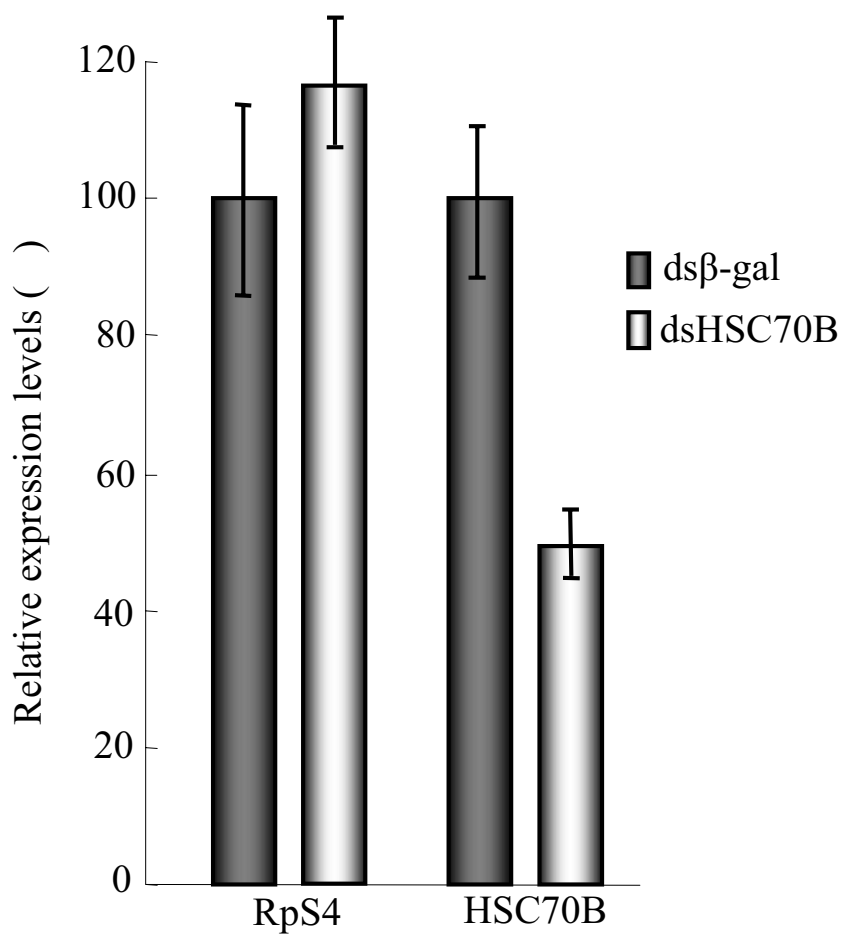

Figure 3

RNA interference efficiency. (A) Expression levels were measured by RT-PCR before (Ctr) and 6 days after the respective dsRNA (RNAi) injection based on agglutinin, HSC70B, and EF-I $\alpha$ and $\beta$-galactosidase (ds $\beta$-gal) as a control. Primers for RTPCR were designed from agglutinin, HSC70B, and EF- I $\alpha$ as well as ribosomal protein gene S7 (RpS7). The expression of RpS7 ( 23 cycles) served as a loading control. (B) The ribosomal protein gene S4 (RpS4) and HSC70B transcript levels (mean \pm SD) were measured by quantitative RT-PCR at 6 days after ONNV-eGFP and dsHSC70B and ds $\beta$-gal injections with 3 biological replicates. Primers for qRT-PCR were designed from RpS4 and HSC70B (Table I). The transcript levels of the loading control (RpS4) did not show significant differences between dsHSC70B and ds $\beta$-gal treatments. However, the HSC70B transcript level in An. gambiae with dsHSC70B injection show an average $58 \%$ reduction of transcript levels compared to that of the control mosquitoes with ds $\beta$-gal treatment (Student's paired t-test, $P=0.0047$ ). 


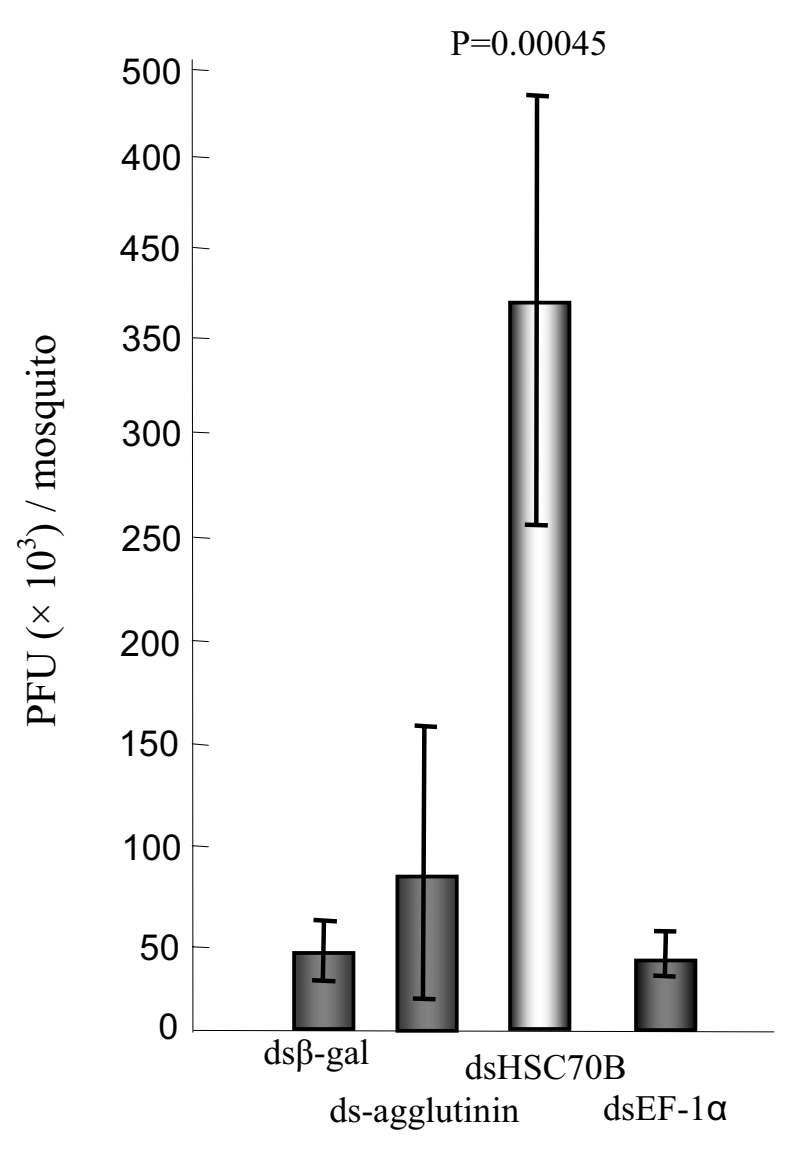

Figure 4

ONNV titers (mean \pm SD) in mosquitoes coinjected with ONNV and the respective dsRNA, including ds $\beta$-gal, ds-agglutinin, dsHSC70B, and dsEF-I $\alpha$. ONNV titers in mosquitoes coinjected with ONNV and dsHSC70B had a statistically significant increase compared to that of coinjection of ONNV and ds $\beta$-gal at 6 days p.i.

(unpaired t-test, $P=0.00045)$. However, ONNV titers of the dsRNAs of the other genes were not significantly different at 6 days p.i. Each data point was generated by five independent mosquitoes which were co-injected by dsRNA and ONNV.

indicate that the low expression levels observed for candidate genes were related to the observed knockdown effect by dsRNAi, and not attributed to variation in sample loading.

\section{Suppression of HSC70B expression by RNAi and higher ONNV infection level decreases the survival rate of An. gambiae}

Co-injection of ONNV and the dsHSC70B significantly shortened the lifespan of adult mosquitoes compared with the co-injected mosquitoes of ONNV and ds $\beta$-gal, causing a significant reduction of survival rates from 7 days p.i. (Fig. 6, P $\leq 0.019$ ). In addition, An. gambiae mosquitoes with down-regulated HSC70B alone lead a reduced survival rate $(\sim 80 \%)$ at 6 days post injection, though it is much less harmful than co-injection of ONNV and dsHSC70B. This suggests that both reduced expression levels of HSC70B gene and increased ONNV infection level synergistically shorten the lifespan of $A n$. gambiae (Fig. 4 and Fig. 6).

\section{Discussion}

In general, the HSP70 family including HSC70B is one of the best studied among the heat shock protein families. The structures of HSP70s and HSC70s are highly conserved in all organisms, from prokaryotes to eukaryotes $[15,16]$. The conserved region of the molecule consists of an N-terminal adenosine triphosphatase domain (ATPase; 400 aa), a substrate-binding domain (SBD; 180 aa), and a carboxyl-terminal domain of variable length and polymorphism [17].

Furthermore, HSC70B, a constitutively induced form of the HSP70 family, was inferred to be located in the cytoplasm [16], and so therefore has may interact with structural and non-structural gene products of ONNV. In fact, from the in vitro study with another alphavirus Sindbis, HSC70 purified from bovine brain inhibits the autoprotease activity of Sindbis capsid protein present in the cytoplasm [18].

Interestingly, the prominent antiviral drug cyclopentenone prostanoids and its derivatives, specifically induced HSP70s and HSC70s in treated Vero and mosquito, Aedes albopictus, cells $[19,20]$. Induction of HSP70s has a cytoprotective effect, interrupting viral replication against a wide range of DNA and RNA viruses including Sindbis $[20,21]$. This antiviral response seems to depend on synthesis of molecular chaperons, which are involved in controlling virus replication in various ways [21]. In addition, recent studies suggested that HSP70 and HSC70 chaperones play a key role in protein quality control, being involved in either folding or degrading of non-native proteins by the ubiquitin-proteasome pathway [22-24].

Alternatively, HSC70B may impede viral replication by modulating other An. gambiae proteins that are critical for viral replication in mosquito cells. Since one of the key roles of HSC70B is to regulate nascent unfolded protein as a chaperone [17], the overexpression of HSC70B may shift metabolism of mosquito cells to suppress viral replication. Thus, HSC70B may indirectly repress nascent protein folding of host factors required for ONNV replication in the mosquito. In contrast, a cDNA microarray study in Ae. aegypti, which is the principal vector of yellow fever and dengue viruses, reported that Sindbis infection significantly down-regulates the mosquito chaperones, HSP62 and HSP80 [25]. Perhaps, viral suppression of host chaperones may be important for propagation of the virus. Pre- 
Table 2: Percentage of mosquitoes displaying eGFP expression in body tissues after coinjection of ONNV-eGFP and dsHSC70B or ds $\beta$ gal at 6 dpi.

\begin{tabular}{cccc}
\hline Treatment & Head & Thorax & Abdomen \\
\hline No dsRNA & $54 \%(20 / 37)$ & $57 \%(21 / 37)$ & $24 \%(9 / 37)$ \\
ds $\beta$-gal & $38 \%(12 / 32)$ & $47 \%(15 / 32)$ & $22 \%(7 / 32)$ \\
dsHSC70B & $70 \%(16 / 23)$ & $87 \%(20 / 23)$ & $65 \%(15 / 23)$ \\
\hline
\end{tabular}

vious studies of the gene products of alphaviruses have shown that these gene products suppress and maneuver host gene expression to favor the viral gene expression system $[26,27]$. With respect to the regulation/repression of host cell gene expression, there may thus be competition between viral and host factors.

Furthermore, the mosquito and virus may compete for the EF- $1 \alpha$ and agglutinin, and this could explain why these proteins are induced upon infection. However, it cannot be ruled out that these knockdown effects may not be sufficient to mimic loss-of-function phenotypes to impair or enhance ONNV replication in An. gambiae, because the RNAi technique cannot completely abolish target transcripts. This lack of complete knockout is expected to allow residual expression of target genes, which could result in protein expression that cannot be phenotypically distinguished from wild type or control groups. Therefore proteomics assays measuring viral protein expression in conjunction with EF-1 $\alpha$ or agglutinin knockdown will provide more definitive pictures for these proteins' role in ONNV replication in An. gambiae.

Suppression of HSC70B by dsRNAi substantially reduced the survival rate of ONNV-infected An. gambiae (Fig. 6). In D. melanogaster, it has been reported that apoptosis affects primarily cells with the lowest level of HSC70s during embryogenesis, suggesting a role for HSC70s in the control of apoptosis [28]. Several studies have shown that HSP70s and HSC70s binding to the anti-apoptotic protein BAG-1 $[29,30]$. It has also been reported that HSC70s interacts with the ecdysone receptor and ultraspiracle (EcR/USP) [31], and that high induction of the chaperones extends longevity in D. melanogaster and in C. elegans [32]. Our data indicates that An. gambiae HSC70B is also important for the survival of mosquitoes during viral infection (Fig. 6).

\section{Conclusion}

In summary, our results demonstrate that HSC70B impede ONNV replication in An. gambiae. The balance between ONNV propagation and HSC70B abundance may determine, at least in part, the level of ONNV replica- tion and cytotoxicity. Understanding the molecular details of HSC70B interaction with structural and nonstructural gene products of ONNV will lead to the elucidation of new approaches to controlling this pathogenic virus. Furthermore, this may suggest new control strategies relying upon the development of transgenic mosquitoes that selectively turn on chaperone genes during arbovirus infection. While molecular mechanisms regarding the regulation of expression of HSP70 genes are relatively well characterized [33], the regulation of HSC70 genes remains to be determined [17].

Our study, in conjunction with the previous microarray experiments, have proven to be effective to identify candidate genes involved in An. gambiae-ONNV interactions at the genome level [7]. In addition to genome-wide gene expression studies, an analysis of tissue-specific gene expression is also important, e.g., midgut, fat body, and salivary glands. The discovery of genes that can provide barriers of ONNV transmission in those mosquito tissues will be beneficial to control the viral infection in humans.

\section{Methods \\ Mosquitoes}

The 4arr strain of An. gambiae was reared at $27^{\circ} \mathrm{C}$ and $80 \%$ relative humidity under a $16 \mathrm{~h}$ light: $8 \mathrm{~h}$ dark photoperiod, as previously described [34]. Adults were supplied with a cotton wool pad soaked in a $10 \%$ sucrose solution ad libitum and fed on anaesthetized guinea pigs once per week for egg production.

\section{Virus}

The SG650, strain of ONNV was obtained from the World Reference Center for Arboviruses at the University of Texas Medical Branch, Galveston, TX. Strain SG650 was isolated from human serum in Uganda in 1996 [35] and has been passed once in Vero cells (GenBank Accession Number AF079456). Stock virus was produced following a single passage in Vero cells maintained at $37^{\circ} \mathrm{C}$ in Leibovitz L-15 medium with $10 \%$ fetal bovine serum (FBS), $100 \mathrm{U} / \mathrm{mL}$ penicillin, and $100 \mu \mathrm{g} / \mathrm{mL}$ streptomycin. Cell supernatant was harvested when $75 \%$ of the cells showed cytopathic effect $(3+\mathrm{CPE})$. Supernatant containing the virus was col- 
A
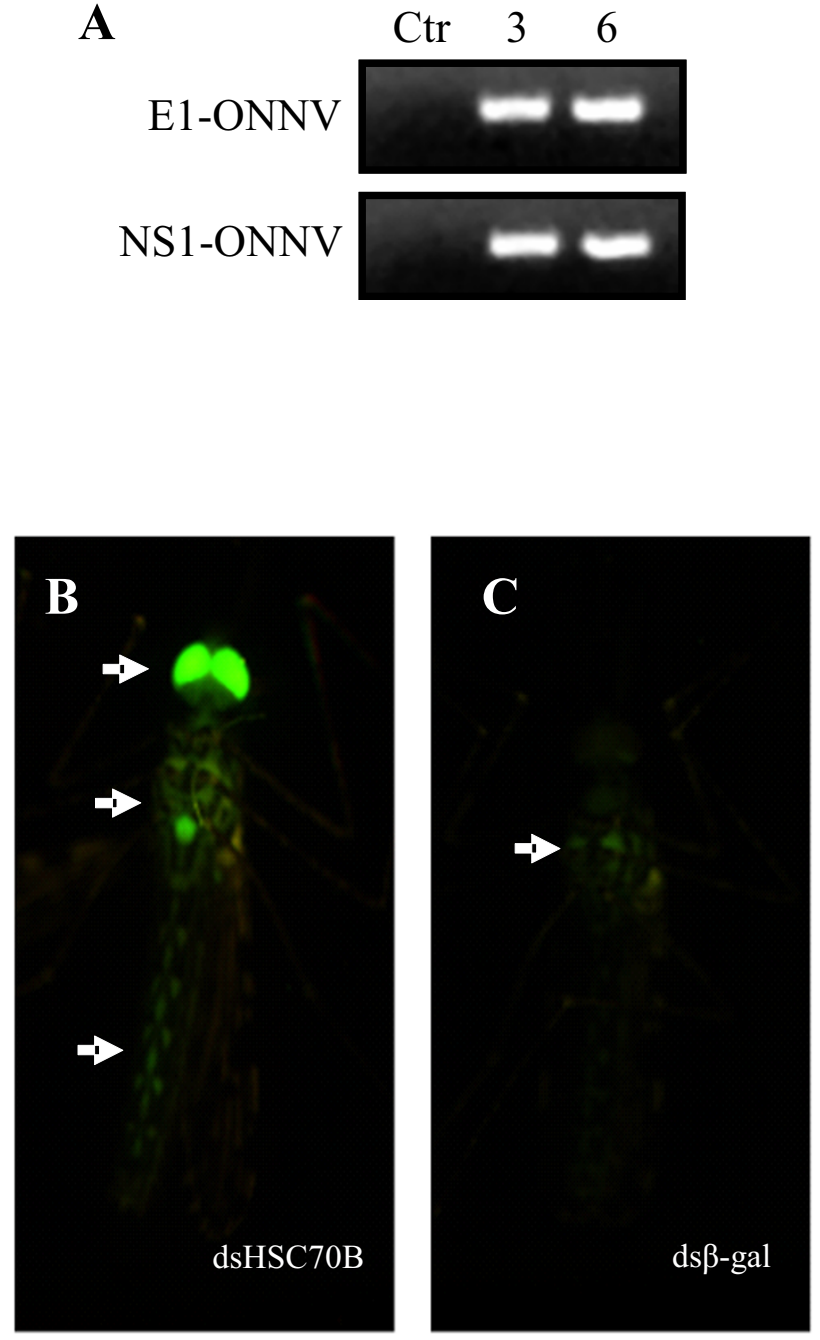

\section{Figure 5}

The phenotype of An. gambiae in response to coinjection of ONNV-eGFP and dsHSC70B. (A) Characterization of viral transcripts of ONNV-eGFP after coinjection with ds $\beta$-gal into adult female An. gambiae (4arr strain). Ctr, 3 and 6 represent transcript profile of the recombinant ONNV with enhanced green fluorescent protein (ONNV-eGFP) before, at 3 and 6 days p.i., respectively. Primers for RT-PCR were designed from the ONNV structural EI gene (EI) and non-structural protein I gene (nsPI) (Table I). The PCR products of the EI and nsPI primer pairs show the expected sizes, $43 \mathrm{I}$ and 440 base pairs, respectively (Table I), indicating the correct expression of ONNV-eGFP in An. gambiae.

(B) The strong expression of GFP in head, thorax, and abdomen of An. gambiae at 6 days after coinjected with dsHSC70B and ONNV-eGFP. (C) The relatively weak expression of GFP in the thorax of An. gambiae at 6 days after coinjected with ds $\beta$-gal and ONNV-eGFP. The arrows indicate the tissues of An. gambiae with GFP expression. lected and titrated. The virus stock contained $2 \times 10^{7}$ plaque-forming units (pfu) $/ \mathrm{ml}$, diluted to $2 \times 10^{6} \mathrm{pfu} / \mathrm{ml}$, aliquoted, and stored at $-80^{\circ} \mathrm{C}$.

\section{Generation of ONNV-eGFP}

The eGFP gene was amplified from pEGFP plasmid (Clontech Laboratories, Inc) using primers Onn-GFP-Asc-F (5'GACCTATGGTGAGCAAGGGCGAGGAGCTGTTC-3')

Onn-GFP-Pac-R (5'-GACCTTTAATTAATTACTTGTACAGCTCGTCCAT-3'). The PCR product was cloned into AscI and PacI sites of infectious clone pONNic-Foy, provided by K. E. Olson and B. D. Foy (Colorado State University, Fort Collins, CO), which was previously modified by replacing the T7 promoter with a SP6 promoter. pONNic-Foy clone was derived from pONN.AP3, developed by Brault and others [36]. Infectious virus from the infectious clone was produced by linearization with Not I, which was in vitro transcribed from the SP6 promoter using the mMESSAGE mMACHINE kit (Ambion, Austin, TX) following the manufacturer's instructions. The RNA was electroporated into BHK-21 cells as previously described [37]. Cell culture supernatant containing virus was harvested, aliquoted, and stored at $-80^{\circ} \mathrm{C}$ when cells showed $3+$ CPE.

\section{dsRNA preparation and coinjection into adult female mosquitoes}

Templates for the preparation of dsRNA for each candidate gene were PCR-derived fragments flanked by two T7 promoter sequences (TAA TAC GAC TCA CTA TAG) (Table 1). Each PCR-derived fragment were sequenced and blasted against the genomic database of An. gambiae [38] to validate the redundancy of the sequence and all confirmed as a unique sequence. Single-stranded RNAs were then synthesized by using the MEGAscript T7 transcription kit (Ambion, Austin, TX) according to the manufacturer's instructions. Annealed dsRNAs were ethanol precipitated and dissolved in injection buffer $(0.1 \mathrm{mM}$ sodium phosphate, $\mathrm{pH} 6.8 ; 5 \mathrm{mM} \mathrm{KCl}$ ). In knockdown experiments, $\sim 0.5 \mu \mathrm{l}$ of a $2: 1 \mathrm{mix}$ of dsRNAs $(2 \mu \mathrm{g} / \mu \mathrm{l})$, ONNV or ONNV-eGFP $\left(\sim 2 \times 10^{6} \mathrm{pfu} / \mathrm{ml}\right)$ were coinjected into the thorax of $\mathrm{CO}_{2}$-anesthetized adult females by using a IM 300 Microinjector (Narishige, Japan). Thus, each mosquito was coinjected with $\sim 3.1 \times 10^{2}$ pfu of virus and $\sim 625$ ng of dsRNA.

\section{Plaque Assays}

Each mosquito was triturated in $1 \mathrm{ml}$ of DMEM, and large particulates were pelleted by centrifugation at 300 r.p.m. and then titrated by standard plaque assay in Vero cells [39]. The plaques were counted and the differences in viral titers were analyzed by Pairwise t-tests. 


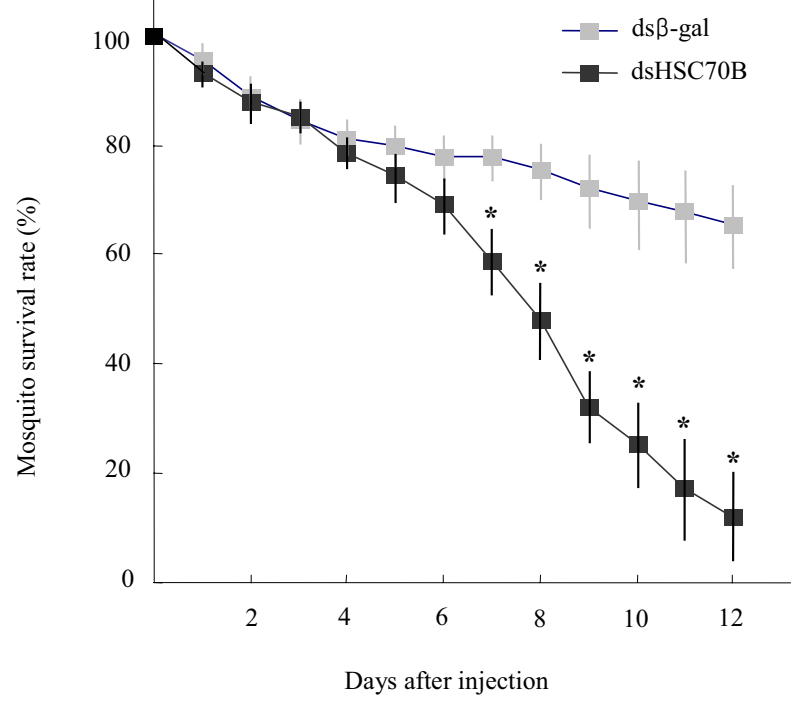

Figure 6

The relative percentages (mean \pm SD) of surviving mosquitoes after the coinjection of ONNV and the respective dsRNA targeting HSC70B and $\beta$-gal (black and gray, respectively). The number of mosquitoes injected represented $100 \%$. Asterisks indicate significant differences in survival (unpaired t-test, $\mathrm{P} \leq 0.019$ ). $\mathrm{N}=6$ groups of $\mathrm{I} 5$ individuals for each data point.

\section{RT-PCR analysis}

Total RNA samples were extracted from three batches of 15 adult female mosquitoes at 6 d.p.i. with ONNV or ONNV-eGFP and the respective dsRNA, using the Trizol Reagent (Invitrogen, CA). To remove genomic DNA contamination, RNA samples were treated with $1.0 \mu \mathrm{l}$ DNase I following the manufacturer's instructions (50-375 units $/ \mu \mathrm{l}$; Invitrogen, CA). For reverse transcription, $5 \mu \mathrm{g}$ of total RNA were reverse transcribed with Superscript III RNase H-reverse transcriptase (Invitrogen). Singlestranded cDNAs of different dilution were amplified by PCR using recombinant Taq DNA polymerase (Invitrogen). To show the RNAi efficiency, primers were made to amplify endogenous agglutinin, EF- $1 \alpha$ and HSC70b genes of An. gambiae. RpS7 gene of An. gambiae was used as an internal control for 23 cycles (Table 1). To characterize the ONNV-eGFP transcript, primers were made to amplify endogenous E1 and NS1 genes of for 23 cycles (Table 1).

\section{Quantitative real-time PCR Analysis (qRT-PCR)}

qRT-PCR was performed using an ABI 7700 Sequence Detection System (Applied Biosystems, CA). Standard curves were generated for each transcript tested using 10fold serial dilutions of An. gambiae genomic DNA ranging from 116 to $0.0116 \mathrm{ng}$ per reaction. All reactions were performed in triplicate in a total volume of $25 \mu \mathrm{l}$ containing $12.5 \mu \mathrm{l}$ of SYBR Green PCR Master Mix, $300 \mathrm{nmol}$ of each primer at the following conditions: $50^{\circ} \mathrm{C}$ for $2 \mathrm{~min}, 95^{\circ} \mathrm{C}$ for $10 \mathrm{~min}$ followed by 50 cycles of denaturation at $95^{\circ} \mathrm{C}$ for $15 \mathrm{~s}$, annealing and extension at $60^{\circ} \mathrm{C}$ for $1 \mathrm{~min}$. RNA samples were extracted from mosquitoes at 6 days p.i. Sequences of gene-specific primer sets are given in Table 1. Statistical significance of differences in the expression of individual genes was determined by using a Student's ttest between the relative transcript values derived from the dsHSC70B-injected and ds $\beta$-gal injected mosquitoes across 3 replicates for each gene.

\section{Survival assay of An. gambiae in co-injection of dsHSC7OB and ONNV}

To evaluate the knockdown effect of HSC70B gene on the survival rate of ONNV infected An. gambiae, 15 females per cohort were intrathoracically co-injected with dsHSC70B and ONNV. For control, 15 females per cohort were intrathoracically coinjected with ds $\beta$-gal and ONNV. Each treated cohort was kept in $8 \mathrm{~cm}$ (diameter) $\times 12 \mathrm{~cm}$ cages with a cotton wool pad soaked a $10 \%$ sucrose solution. The cages were placed at $27^{\circ} \mathrm{C}$ and $80 \%$ relative humidity under a $12 \mathrm{~h}$ light: $12 \mathrm{~h}$ dark photoperiod, and mosquito survival was assessed at 24 hours. Survival was defined as the ability of the mosquito to right itself. Experiments for each of the two groups were replicated six times.

\section{Sources of Sequence Data}

The An. gambiae genome has 10 genes containing the HSP70 domain [40]. Among these, two genes which have a short fragment of HSP70 domain were excluded for further analysis (ENSANGG00000023531, ENSANGG00000023619). The HSP70 gene sequences for Drosophila were obtained from the Berkeley Drosophila Genome Project [41,42].

\section{Multiple Sequence Alignments and Phylogenetic Tree Construction}

Multiple sequence alignments were performed by using ClustalW v1.81 [43]. Phylogenetic trees were constructed by the Neighbor-Joining (NJ) and maximum parsimony (MP) methods, both included in MEGA3 [44]. The accuracy of reconstructed trees was examined by the bootstrap test with 10,000 replications.

\section{Authors' contributions}

CS carried out the study with contributions from YSH, TK and DLV. CS drafted the manuscript with contributions from YSH, KT, DLV, SH and FHC. All authors read and approved the final manuscript.

\section{Acknowledgements}

We wish to thank Dr. K. E. Olson and B. D. Foy for his kind gift of infectious clone pONNic-Foy. This research would not have been possible without the assistance of Dr. Mabel Berois with helpful guide. This project was sup- 
ported by grants ROI-AI44273 from NIH/NIAID to F.H.C. D.L.V. was supported by NIH T32 AI 07536

\section{References}

I. Karabatsos N: Antigenic relationships of group A arboviruses by plaque reduction neutralization testing. Am J Trop Med Hyg 1975, 24(3):527-532.

2. Levinson RS, Strauss JH, Strauss EG: Complete sequence of the genomic RNA of O'nyong-nyong virus and its use in the construction of alphavirus phylogenetic trees. Virology 1990, I75(I): I 10-123.

3. Williams MC, Woodall JP, Corbet PS, Gillett JD: O'nyong-Nyong Fever: An Epidemic Virus Disease in East Africa. 8. Virus Isolations from Anopheles Mosquitoes. Trans R Soc Trop Med Hyg 1965, 59:300-306.

4. Johnson BK: O'nyong-nyong virus disease in The Arboviruses. In Epidemiology and Ecology Volume 3. Edited by: Monath TP. Boca Raton, FL, CRC Press; 1988:pp 217-223.

5. Rwaguma EB, Lutwama J], Sempala SD, Kiwanuka N, Kamugisha J, Okware S, Bagambisa G, Lanciotti R, Roehrig JT, Gubler DJ: Emergence of epidemic O'nyong-nyong fever in southwestern Uganda, after an absence of $\mathbf{3 5}$ years. Emerg Infect Dis 1997, 3(I):77.

6. Posey DL, O'Rourke T, Roehrig JT, Lanciotti RS, Weinberg M, Maloney S: O'Nyong-nyong fever in West Africa. Am J Trop Med Hyg 2005, 73(I):32.

7. Sim C, Hong YS, Vanlandingham DL, Harker BW, Christophides GK, Kafatos FC, Higgs S, Collins FH: Modulation of Anopheles gambiae gene expression in response to o'nyong-nyong virus infection. Insect Mol Biol 2005, I4(5):475-48I.

8. Morimoto RI, Santoro MG: Stress-inducible responses and heat shock proteins: new pharmacologic targets for cytoprotection. Nat Biotechnol 1998, I 6(9):833-838.

9. Joshi RL, Ravel JM, Haenni AL: Interaction of turnip yellow mosaic virus Val-RNA with eukaryotic elongation factor EFI [alpha]. Search for a function. Embo J | 986, 5(6): | |43- | | 48.

10. Barrera I, Schuppli D, Sogo JM, Weber H: Different mechanisms of recognition of bacteriophage $Q$ beta plus and minus strand RNAs by $Q$ beta replicase. I Mol Biol 1993, 232(2):5|2-52|.

I I. Blackwell JL, Brinton MA: Translation elongation factor-I alpha interacts with the 3 ' stem-loop region of West Nile virus genomic RNA. J Virol 1997, 7 I(9):6433-6444.

12. Brinton MA: Host factors involved in West Nile virus replication. Ann N Y Acad Sci 2001, 95 I:207-219.

13. Kaariainen L, Ahola T: Functions of alphavirus nonstructural proteins in RNA replication. Prog Nucleic Acid Res Mol Biol 2002, 7I:187-222.

14. Richman AM, Dimopoulos G, Seeley D, Kafatos FC: Plasmodium activates the innate immune response of Anopheles gambiae mosquitoes. Embo J 1997, 16(20):61 14-6II9.

15. Gupta RS, Golding GB: Evolution of HSP70 gene and its implications regarding relationships between archaebacteria, eubacteria, and eukaryotes. J Mol Evol 1993, 37(6):573-582.

16. Boorstein WR, Ziegelhoffer T, Craig EA: Molecular evolution of the HSP70 multigene family. J Mol Evol I994, 38(I): I-I7.

17. Kiang JG, Tsokos GC: Heat shock protein $70 \mathrm{kDa}$ : molecular biology, biochemistry, and physiology. Pharmacol Ther 1998, 80(2): |83-20|.

18. Ryan C, Stevens TH, Schlesinger MJ: Inhibitory effects of HSP70 chaperones on nascent polypeptides. Protein Sci 1992, I (8):980-985.

19. Burlandy FM, Ferreira DF, Rebello MA: Inhibition of vesicular stomatitis virus replication by prostaglandin $A I$ in Aedes albopictus cells. Z Naturforsch [C] 2004, 59(I-2): I27-I31.

20. Amici C, Giorgi C, Rossi A, Santoro MG: Selective inhibition of virus protein synthesis by prostaglandin AI: a translational block associated with HSP70 synthesis. J Virol I994, 68( I I):6890-6899.

21. Mastromarino P, Conti C, Petruzziello R, De Marco A, Pica F, Santoro MG: Inhibition of Sindbis virus replication by cyclopentenone prostaglandins: a cell-mediated event associated with heatshock protein synthesis. Antiviral Res 1993, 20(3):209-222.
22. Cyr DM, Hohfeld J, Patterson C: Protein quality control: U-boxcontaining E3 ubiquitin ligases join the fold. Trends Biochem Sci 2002, 27(7):368-375.

23. Ellgaard L, Helenius A: Quality control in the endoplasmic reticulum. Nat Rev Mol Cell Biol 2003, 4(3): I8I-191.

24. McClellan AJ, Tam S, Kaganovich D, Frydman J: Protein quality control: chaperones culling corrupt conformations. Nat Cell Biol 2005, 7(8):736-74I.

25. Sanders HR, Foy BD, Evans AM, Ross LS, Beaty BJ, Olson KE, Gill SS: Sindbis virus induces transport processes and alters expression of innate immunity pathway genes in the midgut of the disease vector, Aedes aegypti. Insect Biochem Mol Biol 2005, 35( I I): I293-I307.

26. Strauss $\mathrm{JH}$, Strauss EG: The alphaviruses: gene expression, replication, and evolution. Microbiol Rev 1994, 58(3):491-562.

27. Kaariainen L, Ranki M: Inhibition of cell functions by RNA-virus infections. Annu Rev Microbiol 1984, 38:91-109.

28. de la Rosa EJ, Vega-Nunez E, Morales AV, Serna J, Rubio E, de Pablo F: Modulation of the chaperone heat shock cognate 70 by embryonic (pro)insulin correlates with prevention of apoptosis. Proc Natl Acad Sci U S A 1998, 95(I 7):9950-9955.

29. Takayama S, Bimston DN, Matsuzawa S, Freeman BC, Aime-Sempe C, Xie Z, Morimoto RI, Reed JC: BAG-I modulates the chaperone activity of Hsp70/Hsc70. Embo J I997, I 6( I 6):4887-4896.

30. Takayama S, Krajewski S, Krajewska M, Kitada S, Zapata JM, Kochel K, Knee D, Scudiero D, Tudor G, Miller GJ, Miyashita T, Yamada M, Reed JC: Expression and location of Hsp70/Hsc-binding antiapoptotic protein BAG-I and its variants in normal tissues and tumor cell lines. Cancer Res 1998, 58( I 4):3 I I6-3 I 3 I.

31. Arbeitman MN, Hogness DS: Molecular chaperones activate the Drosophila ecdysone receptor, an $\mathbf{R X R}$ heterodimer. Cell 2000, I0I(I):67-77.

32. Tatar M, Khazaeli AA, Curtsinger JW: Chaperoning extended life. Nature 1997, 390(6655):30.

33. Morimoto RI, Kroeger PE, Cotto J]: The transcriptional regulation of heat shock genes: a plethora of heat shock factors and regulatory conditions. Exs 1996, 77:139-163.

34. Higgs S: Care, maintenance, and experimental infection of mosquitoes. In The Biology of Disease Vectors Edited by: Marquardt WC, Kondratieff B, C.G. M, Freier J, Hagedorn HH, Black WIII, James AA, Hemingway J, Higgs S. Niwot, CO , Elsevier Academic Press; 2004:pp. 733-739.

35. Lanciotti RS, Ludwig ML, Rwaguma EB, Lutwama JJ, Kram TM, Karabatsos N, Cropp BC, Miller BR: Emergence of epidemic O'nyong-nyong fever in Uganda after a 35-year absence: genetic characterization of the virus. Virology 1998, 252(I):258-268.

36. Brault AC, Foy BD, Myles KM, Kelly CL, Higgs S, Weaver SC, Olson $\mathrm{KE}$, Miller BR, Powers AM: Infection patterns of o'nyong nyong virus in the malaria-transmitting mosquito, Anopheles gambiae. Insect Mol Biol 2004, I3(6):625-635.

37. Vanlandingham DL, Tsetsarkin K, Klingler KA, Hong C, McElroy KL, Lehane MJ, Higgs S: Determinants of vector specificity of o'nyong nyong and chikungunya viruses in Anopheles and Aedes mosquitoes. Am J Trop Med Hyg 2006, 74(4):663-669.

38. The NCBI BLAST Database [http://www.ncbi.nlm.nih.gov/ BLAST/]

39. Myles KM, Pierro DJ, Olson KE: Deletions in the putative cell receptor-binding domain of Sindbis virus strain MREI6 E2 glycoprotein reduce midgut infectivity in Aedes aegypti. J Virol 2003, 77( 16):8872-8881.

40. Vectorbase [http://agambiae.vectorbase.org/SequenceData/ Genome/]

41. The Berkeley Drosophila Genome Database [http://www.fruit fly.org/]

42. Adams MD, Celniker SE, Holt RA, Evans CA, Gocayne JD, Amanatides PG, Scherer SE, Li PW, Hoskins RA, Galle RF, George RA, Lewis SE, Richards S, Ashburner M, Henderson SN, Sutton GG, Wortman JR, Yandell MD, Zhang Q, Chen LX, Brandon RC, Rogers YH, Blazej RG, Champe M, Pfeiffer BD, Wan KH, Doyle C, Baxter EG, Helt G, Nelson CR, Gabor GL, Abril JF, Agbayani A, An HJ, Andrews-Pfannkoch C, Baldwin D, Ballew RM, Basu A, Baxendale J, Bayraktaroglu L, Beasley EM, Beeson KY, Benos PV, Berman BP, Bhandari D, Bolshakov S, Borkova D, Botchan MR, Bouck J, Brokstein P, Brottier P, Burtis KC, Busam DA, Butler H, Cadieu E, Center A, Chandra I, Cherry JM, Cawley S, Dahlke C, Davenport LB, Davies P, de Pablos B, Delcher A, 
Deng Z, Mays AD, Dew I, Dietz SM, Dodson K, Doup LE, Downes M, Dugan-Rocha S, Dunkov BC, Dunn P, Durbin KJ, Evangelista CC, Ferraz C, Ferriera S, Fleischmann W, Fosler C, Gabrielian AE, Garg NS, Gelbart WM, Glasser K, Glodek A, Gong F, Gorrell JH, Gu Z, Guan P, Harris M, Harris NL, Harvey D, Heiman TJ, Hernandez JR, Houck J, Hostin D, Houston KA, Howland TJ, Wei MH, Ibegwam C, Jalali M, Kalush F, Karpen GH, Ke Z, Kennison JA, Ketchum KA, Kimmel BE, Kodira CD, Kraft C, Kravitz S, Kulp D, Lai Z, Lasko P, Lei Y, Levitsky AA, Li J, Li Z, Liang Y, Lin X, Liu X, Mattei B, Mclntosh TC, McLeod MP, McPherson D, Merkulov G, Milshina NV, Mobarry C, Morris J, Moshrefi A, Mount SM, Moy M, Murphy B, Murphy L, Muzny DM, Nelson DL, Nelson DR, Nelson KA, Nixon K, Nusskern DR, Pacleb JM, Palazzolo M, Pittman GS, Pan S, Pollard J, Puri V, Reese MG, Reinert K, Remington K, Saunders RD, Scheeler F, Shen H, Shue BC, SidenKiamos I, Simpson M, Skupski MP, Smith T, Spier E, Spradling AC, Stapleton M, Strong R, Sun E, Svirskas R, Tector C, Turner R, Venter E, Wang $A H$, Wang $X$, Wang ZY, Wassarman DA, Weinstock GM, Weissenbach J, Williams SM, WoodageT, Worley KC, Wu D, Yang S, Yao QA, Ye J, Yeh RF, Zaveri JS, Zhan M, Zhang G, Zhao Q, Zheng L, Zheng XH, Zhong FN, Zhong W, Zhou X, Zhu S, Zhu X, Smith HO, Gibbs RA, Myers EW, Rubin GM, Venter JC: The genome sequence of Drosophila melanogaster. Science 2000, 287(546I):2185-2| 95 .

43. Thompson JD, Higgins DG, Gibson TJ: CLUSTAL W: improving the sensitivity of progressive multiple sequence alignment through sequence weighting, position-specific gap penalties and weight matrix choice. Nucleic Acids Res 1994, 22(22):4673-4680.

44. Kumar S, Tamura K, Nei M: MEGA: Molecular Evolutionary Genetics Analysis software for microcomputers. Comput Appl Biosci 1994, 10(2):|89-19|.

\section{Publish with Bio Med Central and every scientist can read your work free of charge}

"BioMed Central will be the most significant development for disseminating the results of biomedical research in our lifetime. "

Sir Paul Nurse, Cancer Research UK

Your research papers will be:

- available free of charge to the entire biomedical community

- peer reviewed and published immediately upon acceptance

- cited in PubMed and archived on PubMed Central

- yours - you keep the copyright

Submit your manuscript here:

http://www.biomedcentral.com/info/publishing_adv.asp
BioMedcentral 